\title{
Diagnosis of faults in the bearings by electrical measures and numerical simulations
}

\author{
Charles Machado ${ }^{1, a}$, Mohamed Guessasma ${ }^{1}$, Emannuel Bellenger ${ }^{1}$, \\ Khaled Bourbatache ${ }^{2}$, VAléry Bourny ${ }^{1}$ and JÉrôme Fortin ${ }^{1}$ \\ 1 Laboratoire des Technologies Innovantes (LTI EA 3899), UPJV, 48 rue Raspail, 02100 Saint-Quentin, France \\ 2 LGCGM, INSA de Rennes, 35000 Rennes, France
}

Received 7 January 2014, Accepted 15 May 2014

\begin{abstract}
This project represents a straight continuation of the research activities of the LTI and which helped to simulate and understand the electrical response of multi-contact systems. In order to make further progress in the electrical modeling, the electrical transfer model is applied to the case of bearing operation. The 2D modeling by discrete elements reproduces the dynamic bearing. The method proposes to take measurements of electrical resistance on bearings to detect defects. This is an original method that stems from work published in MSSP. Indeed, while many studies have been conducted on monitoring bearing defects by analyzing vibration signals, the use of localized electrical measurements on bearings is a promising approach, not yet exploited. The introduction of the first defects, as a local breaking of mechanical contact causes periodic disturbances in the calculation of the electrical resistance. One of our objectives is to characterize the defects position and the size of the defects, by analyzing disturbances in the electrical measurement.
\end{abstract}

Key words: Bearing defects / diagnosis / electrical measurement / discrete elements / statistical indicators

\section{Introduction}

Rolling bearings are one of the most important and frequently encountered components in the industrial rotating machineries. Statistical studies show that these bearings are considered as critical mechanical components. According to different sources, they represent between $40 \%$ and $50 \%$ of misfunction in rotating machineries, affecting the machine availability and the surrounding systems. An undetected defect in a bearing can lead to catastrophic failure for the rotating machines. Therefore, in order to ensure the industrial systems availability and the safety of goods and persons, the monitoring and diagnosis of bearing defects have to be considered with prime importance and the challenges in terms of productivity are non-negligible. Thus, defect detection in rolling element bearings has led to extensive research. Different experimental methods have been proposed for detection and diagnosis of bearing defects which may be broadly classified as vibration and acoustic measurements, temperature measurements, defect signatures in the stator current motors, wear debris and lubricant analysis. Among these, vibration measurements are the most widely used.

\footnotetext{
${ }^{a}$ Corresponding author: charles.machado@u-picardie.fr
}

Many researches have been published with several approaches [1] for the detection and diagnosis of bearing defects by vibration measurements along with signal processing techniques in both time and frequency domains. Vibration in the time domain can be measured through parameters such as the overall Root-Mean-Square level of acceleration, crest factor, probability density of acceleration, kurtosis $[2,3]$. Our goal is to provide a new tool for predictive maintenance which will operate the local electrical measurements (current, voltage, impedance) to monitor the mechanical state of the bearing. This means, a low current flows through the bearing by the rolling elements and measures for an electrical resistance in real time. An experimental prototype shows the feasibility of this method (Fig. 1).

We seek to analyze the electrical signatures observed on the red graph (Fig. 1), to characterize the defect in position and in size. We have developed a numerical approach based on the discrete elements method $[4,5]$ to model the phenomena of information transfer between the mechanical state and the electrical measurement [6]. The assumptions are that the components of ball bearing are rigid and the lubricant has not been considered. In this paper, we are going to present the first results for this innovative approach. We have introduced various defects 

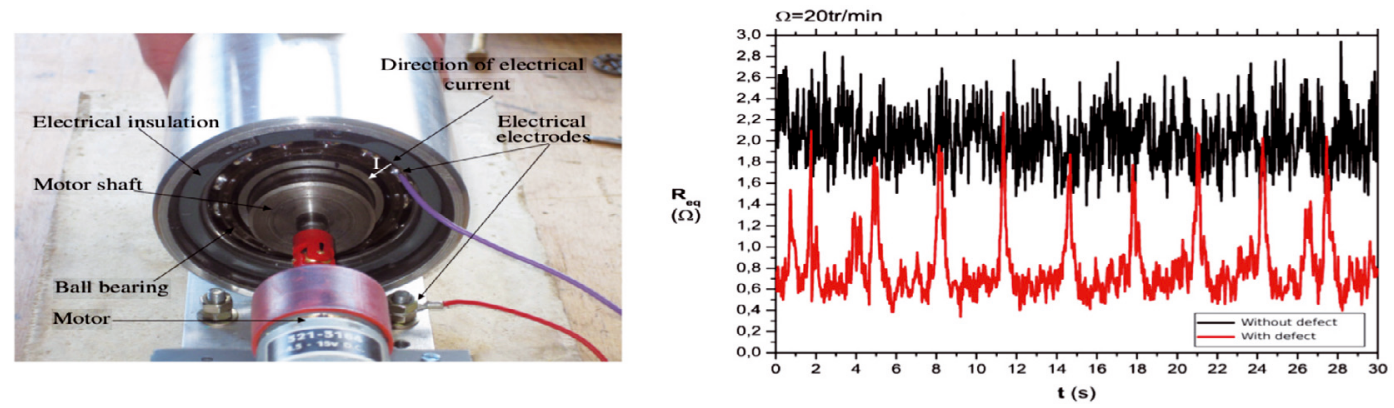

Fig. 1. Experimental prototype and experimental electrical resistance with and without defect.

with different sizes and calculated statistical indicators. The following development consitutes a feasibility study which will be refined in the future.

\section{Electromechanical modeling in multi-contact systems}

The complex mechanical behavior of the ball bearing requires working with numerical methods. The electromechanical coupling implies to know an electrical transfer model depending of the mechanical configuration.

\subsection{Electromechanical modeling}

The 2D modeling of the bearing by discrete elements method (DEM) considers the ball bearing as a turnover multi-contact system consisting with a set of particles subjected to mechanical loading [4-7]. The DEM is based on the Non Smooth Contact Dynamics (NSCD) $[8,9]$ which considers non-deformable and non-penetrable particles. The NSCD is a branch of the DEM that accurately allows for modeling the dynamic effects with a large time discretization. Contact detection allows for studying the phenomena of energy transfer.

Generally, the electrical transfer in multi-contact systems depends on the intrinsic mechanical and electrical properties of materials, on the number and shape of particles and on the contact number, as suggested by our recent researches [6]. Electrical response depends also on the mechanical loading applied to multi-contact systems. Indeed, the paths of electrical current follow the force chains. The bearing is considered as a particular type of multi-contact system, where the coordination number is constant. Moreover, a comparison between the electrical behavior of granular materials and ball bearing shows that their electrical responses are very similar. The electrical transfer mostly depends with the local phenomena activated at contact surfaces. The electrical conductance model takes into account intrinsic mechanical quantities and electric properties of materials. In this study, we assumed that the medium temperature is constant, the oxide layer on the surface of particles and the lufrification are not taken into account. The effect of roughness is neglected. The contact is perfect and the electrical transfer is described by a linear Ohm law. The formulation of the electrical problem is based on the contact network of a multi-contact system. The main idea is to use the first Kirchhoff law and the Ohm's law to build the problem to solve. The electrical potentials at each contact point are the unknowns of the electrical problem.

The electrical conductance between two contact surfaces $S_{i}$ and $S_{j}$ (contact surfaces at contact points $i$ and $j$ respectively) located on the surface of a homogeneous spherical particle $k$ (Fig. 2) is given by:

$$
C_{i j}^{k}=\frac{1}{R_{i j}^{k}}=\frac{\gamma S_{i} S_{j}}{2 V_{\mathrm{b}}}(1-\cos \theta)
$$

where $\gamma$ is the electrical conductivity of the material, $V_{\mathrm{b}}$ is the volume of particle, $\theta$ is the angle formed by the points $i, j$ and the center of particle (Fig. 4). The coupling between the mechanical and electrical computation is carried out by Hertz's theory [10]. In the ball bearings context, the ball-races contact surface is elliptical. This contact surface is computed by using the Greenwood's model [10] which allows to compute the semi-axes $a$ and $b$ of the elliptical contact (Fig. 3). We consider $f_{\mathrm{n}}$ the normal contact force at the contact point by using the Hertz's theory given by:

$$
c=\left(\frac{3 f_{\mathrm{n}} r_{\mathrm{eff}}}{4 E_{\mathrm{eff}}}\right)^{\frac{1}{3}}, \quad c^{2}=a b
$$

where $E_{\text {eff }}$ denotes the effective Young modulus given by formula $\frac{1}{E_{\text {eff }}}=\frac{2\left(1-\nu^{2}\right)}{E}$, where $E$ and $\nu$ are respectively the Young's modulus and Poisson's ratio of the material. $r_{\text {eff }}$ is the effective radius for an elliptical contact given by the following expression:

$$
r_{\mathrm{eff}}=\left(A B\left(\frac{A+B}{2}\right)\right)^{\frac{-1}{3}}
$$

where $A$ and $B$ are given in the case of contact ball-outer (inner) races by:

$$
A=\frac{1}{R_{\mathrm{b}}}+\frac{1}{R_{\text {ext(inn })}^{c}}, B=\frac{1}{R_{\mathrm{b}}}+\frac{1}{R_{\text {ext(inn })}}
$$

where $R_{\mathrm{b}}$ is the radius of the ball in contact, $R_{\text {ext(inn) }}$ is the radius of outer (inner) race of the ball bearing 


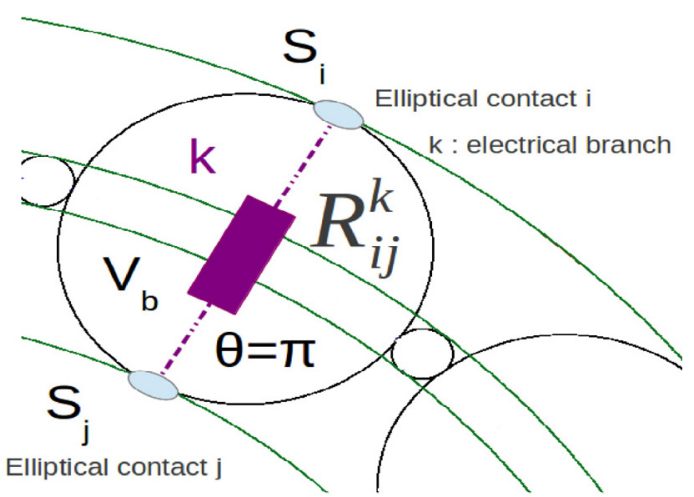

Fig. 2. Electrical conductance.

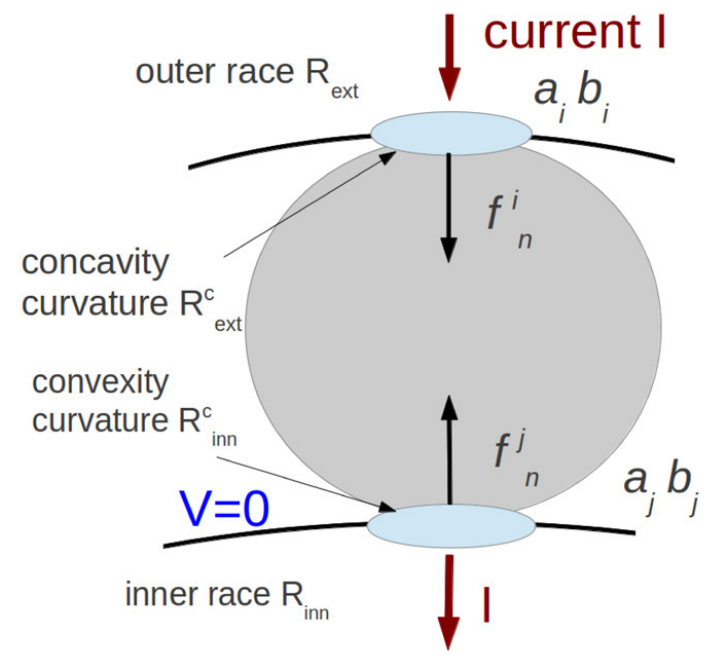

Fig. 3. Elliptical contacts.

and $R_{\text {ext(inn) }}^{\mathrm{c}}$ is the radius of curvature of the outer (inner) race. $R_{\text {ext(inn) }}^{\mathrm{c}}$ is positive or negative if the surface is concave or convex respectively. The semi axes of the elliptical contact surface are determined by the following approximation [10]:

$$
c^{2}=a b, \quad \frac{a}{b}=\left(\frac{A}{B}\right)^{\frac{2}{3}}
$$

The contact surfaces are given by formula $S_{i(j)}=$ $\pi a_{i(j)} b_{i(j)}=\pi c_{i(j)}^{2}$.

The electromechanical coupling is implemented in the DEM MULTICOR software [5-7], the corresponding algorithm is given by Figure 4 .

In this study, we consider a weak electromechanical coupling, the electrical computation depends on the mechanical solution. The MULTICOR software determines positions, velocities of all particles and the contact reactions in each contact point. The electrical conductance is computed by equation (1). Knowledge of normal stresses $\mathrm{ball} / \mathrm{ring}$, at time $t$, is sufficient to determine the electrical resistance of a rolling element. The total equivalent resistance is determined by the matching of resistors in parallel. The matrix system is built for the determination

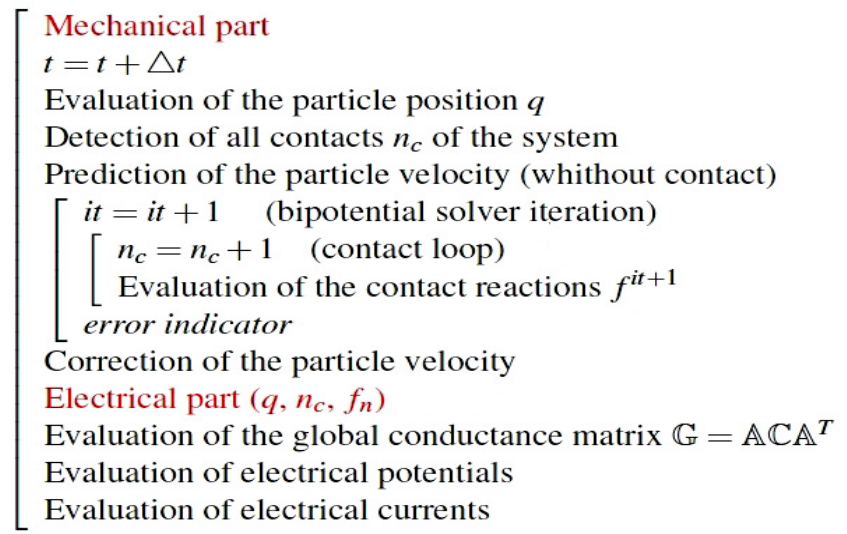

Fig. 4. Algorithm of electromechanical resolution.

Table 1. Geometrical characteristics of ball bearing.

\begin{tabular}{cccccc}
\hline$D_{\text {ext }}$ & $D_{\text {inn }}$ & $D_{\mathrm{b}}$ & $N_{\mathrm{b}}$ & $R_{\text {ext }}^{\mathrm{c}}$ & $R_{\text {inn }}^{\text {c }}$ \\
\hline $73.2 \mathrm{~mm}$ & $48 \mathrm{~mm}$ & $12.6 \mathrm{~mm}$ & 13 & $6.65 \mathrm{~mm}$ & $6.55 \mathrm{~mm}$ \\
\hline
\end{tabular}

of electrical potentials at each contact point. This system is solved by the Gauss method. The proposed electrical discrete element method (EDEM) have been implemented and validated in the static case in $1 \mathrm{D}$ and $2 \mathrm{D}[6]$.

\subsection{Geometrical characteristics of the bearing}

The real bearing (SKF 6208) is composed with 13 rolling elements (balls). An insulating cage keeps a constant gap between balls. The assembly is held between the clamped outer ring and the rotatable inner ring. An analogy between the real system and the numerical model is presented in Figure 5. The rolling elements are steel balls and the cage is represented by insulating balls.

The geometrical characteristics of the simulated ball bearing (Fig. 5 ) are given in Table 1 where $D_{\text {ext }}$ and $D_{\text {inn }}$ are the diameters of outer and inner races respectively (Fig. 5). $D_{\mathrm{b}}$ and $N_{\mathrm{b}}$ represent the diameter and number of rolling balls. $R_{\text {ext }}^{\mathrm{c}}$ and $R_{\text {inn }}^{\mathrm{c}}$ are the curvature radius of outer and inner races respectively.

The electrical conductance model, given by (1) allows contact between steel (race) and steel (ball), the effect of lubricant, oxide layer and the roughness are not taken into account (perfect Hertz's contact). To describe the dynamics of rotation, we introduce the angular velocity $\omega$ of the inner race. The outer race receives an isotropic force $F$, directed towards the center of the race. This force can be likened to "a tightening". A friction coefficient (steel-steel) $\mu$ implies a sliding phase. In this model, for each ball, four contacts are possible with its neighbours (inner race, outer race and cage). Even if these assumptions seem very restrictive, work on metal granular media have shown that the proposed model (1) was enough to compare with experimental measurements. It's always possible to complete this model, considering the effect of lubricant (depending on thickness). This argument is 


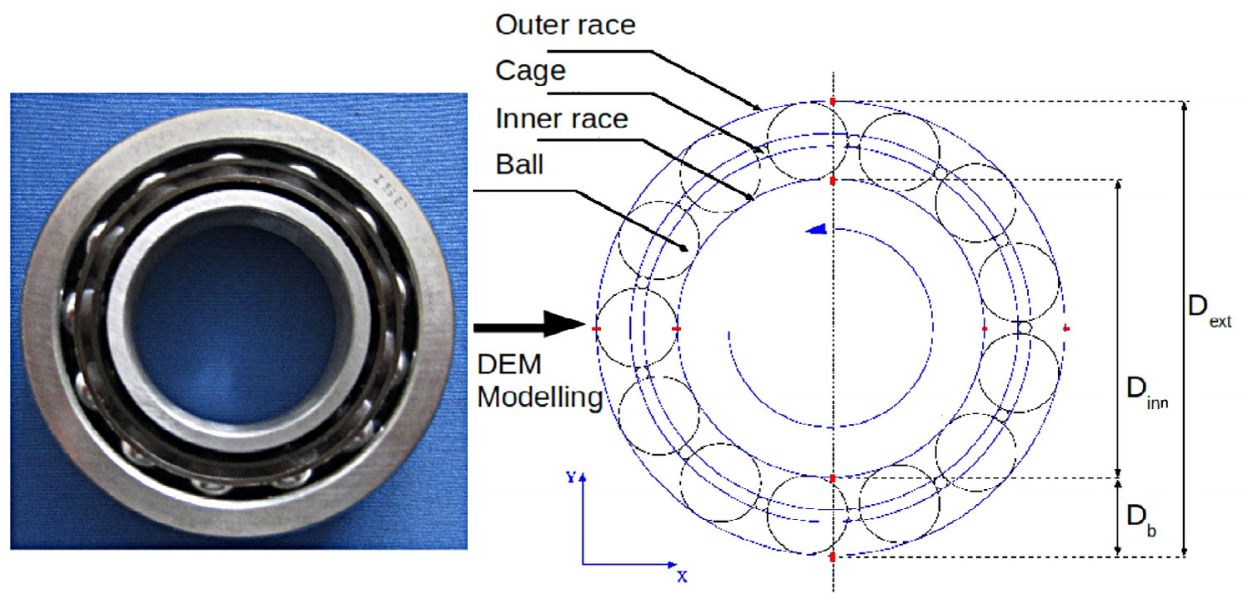

Fig. 5. DEM model of ball bearing.
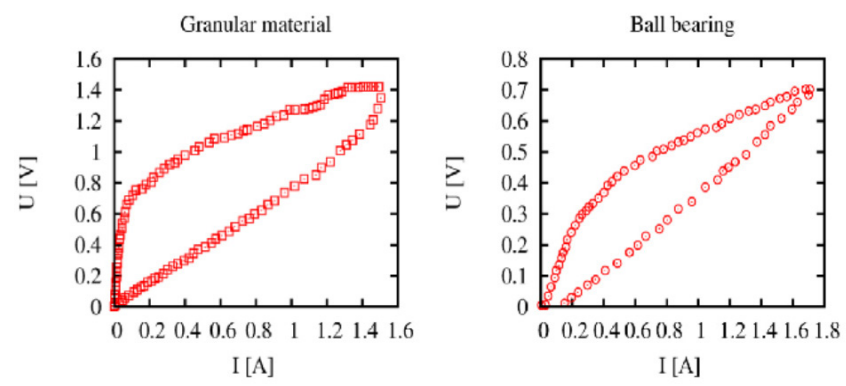

Fig. 6. Experimental comparison of static responses.

Table 2. Mechanical and electrical properties.

\begin{tabular}{cccc}
\hline$E$ & $\nu$ & $\mu$ & $\gamma$ \\
\hline $200 \mathrm{Gpa}$ & 0.3 & 0.15 & $5.88 \times 10^{7}{\mathrm{~S} . \mathrm{m}^{-1}}$ \\
\hline
\end{tabular}

reinforced by the fact that experimentally the electrical behavior of the two systems are similar.

The mechanical properties and electrical properties depend on the considered materials. In the case of the studied bearing (Fig. 5), we have the following properties (Tab. 2).

\section{Numerical modeling of a healthy bearing}

The numerical results in the case of a healthy bearing are a reference. These calculations are used to verify the modeling of bearing operation and to compare with the case of bearing with defects. All simulations are carried out for the same initial conditions (Fig. 7). The fixed outer ring is compressed by the force $F$. The input current $I$ is set at $0.1 \mathrm{~A}$, distributed on contact ball-outer race, weighted in relation to the normal reactions $f_{\mathrm{n}}$. The inner race receives the operating speed $\omega=500 \mathrm{rad} . \mathrm{s}^{-1}$ (about $4500 \mathrm{rpm}$ ). Each ball-inner race contact are at zero potential $\left(V_{\mathrm{inn}}=0\right)$. Electrical potentials $V_{\text {out }}$ at each ball-outer race contact are the unknowns of the electrical problem. $V_{\text {out }}$ are determined at each time step with the

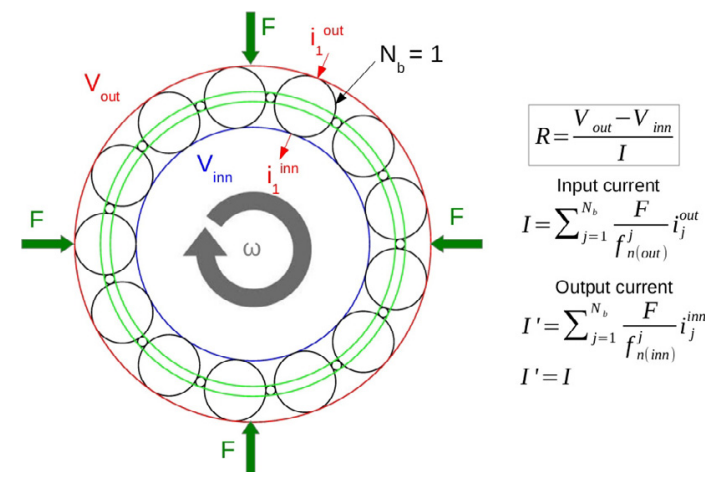

Fig. 7. Initial operating conditions.

conductance model (1), using Kirchhoff's laws and the Ohm's law. The time step calculation is $10^{-6} \mathrm{~s}$. Thanks to the simulation, the electrical resistance can be determined for each time step, using the mechanical configuration.

\subsection{Simulation of a healthy bearing}

The numerical results obtained from the simulation are represented in Figure 8.

Figure $8 \mathrm{a}$ shows that the ball velocity is constant (modulus). These velocities induce inclusion of a centrifugal force, shown in Figure 8b, between the inner race and the outer race. The electrical potential (Fig. 8c) shows that the current only passes through the rolling elements.

The first observation in Figure 9a suggests that contacts are never lost. We can also note that the system goes by a sliding phase $(\tau \sim 0.004 \mathrm{~s})$ before establishing its stationary regime (Figs. 9b-d). After the sliding phase, normal reactions reach their limits. The outer race limit reaction is higher than the inner race limit reaction due to the contribution of the centrifugal force (Fig. 9c). For $t>\tau$, the electrical resistance is about $1.39 \Omega$ (Fig. $9 \mathrm{~b}$ ). 

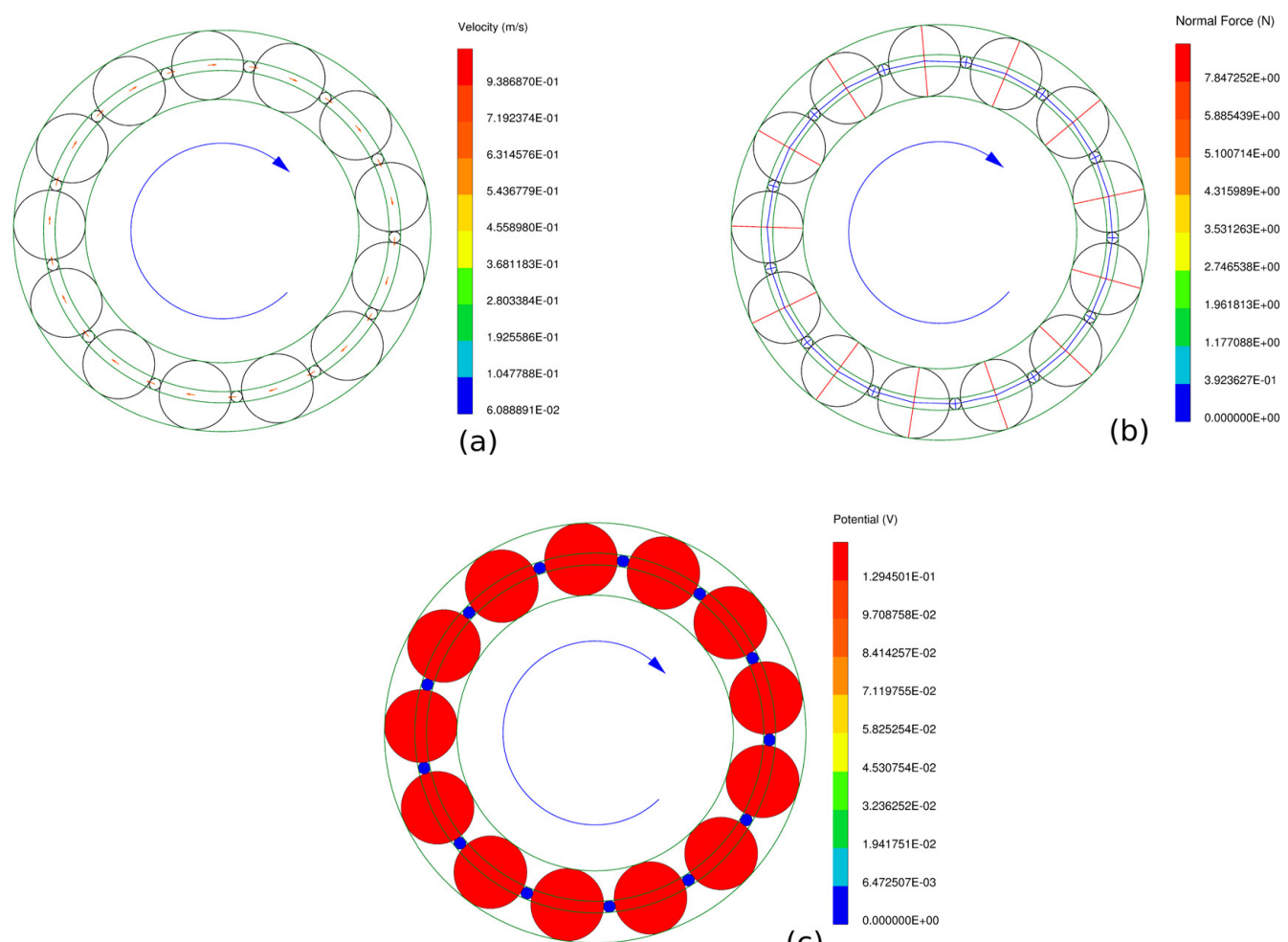

(c)

Fig. 8. Numerical results with DEM ball bearing model. (a) Velocity (b) Contact force distribution (c) Electrical potential distribution.
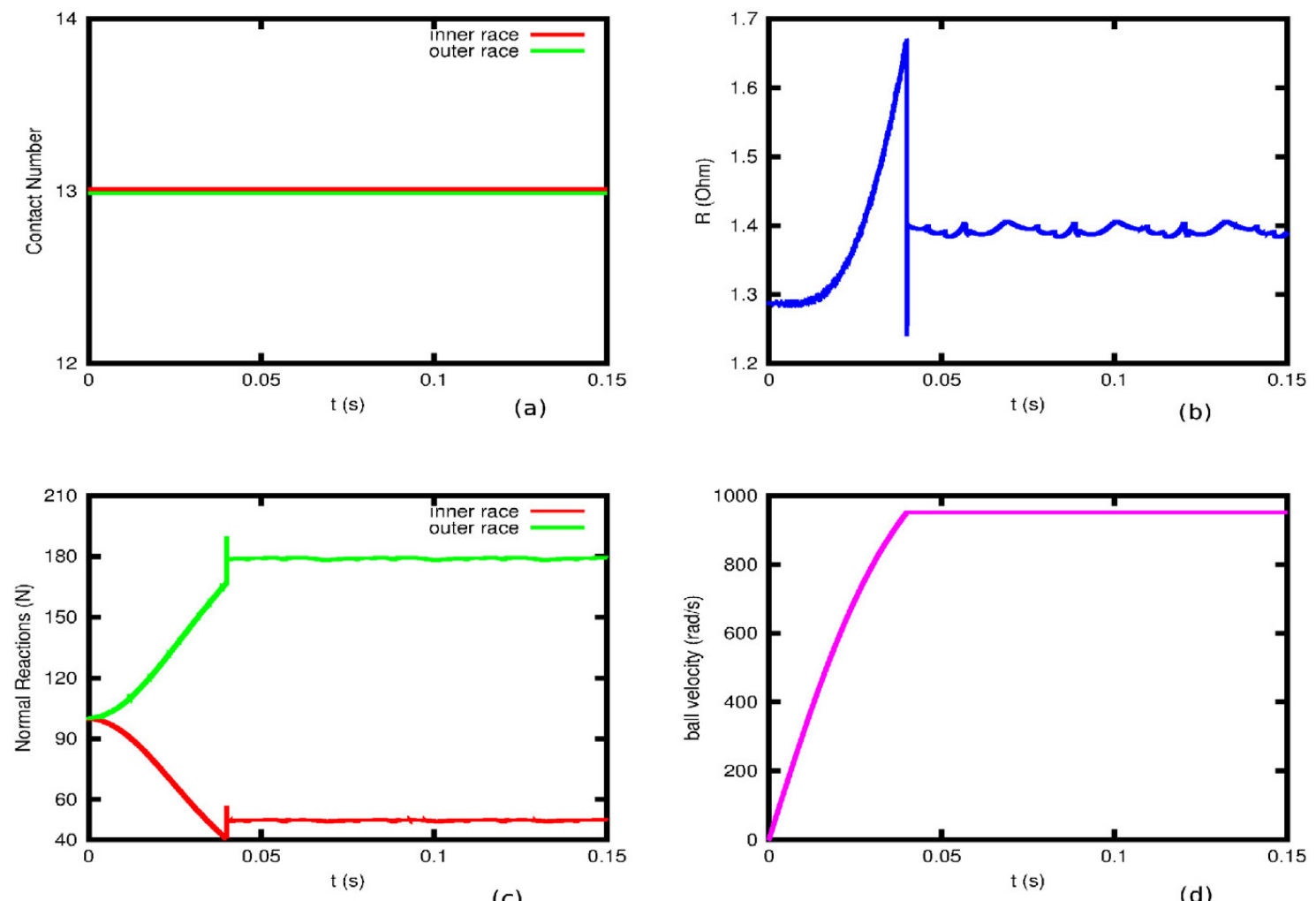

Fig. 9. Numerical observations of the healthy bearing according to time: (a) Contact number. (b) Electrical resistance. (c) Normal reactions. (d) Ball velocity. 
Table 3. Bearing defects agreement.

\begin{tabular}{ccccc}
\hline Defect location & Ball & Cage & Inner race & Outer race \\
\hline Agreement & BSF & FTF & BPFI & BPFO \\
\hline Meaning & $\begin{array}{c}\text { Ball spin } \\
\text { frequency }\end{array}$ & $\begin{array}{c}\text { Fundamental } \\
\text { train frequency }\end{array}$ & $\begin{array}{c}\text { Ball passing } \\
\text { frequency inner }\end{array}$ & $\begin{array}{c}\text { Ball passing } \\
\text { frequency outer }\end{array}$ \\
\hline
\end{tabular}

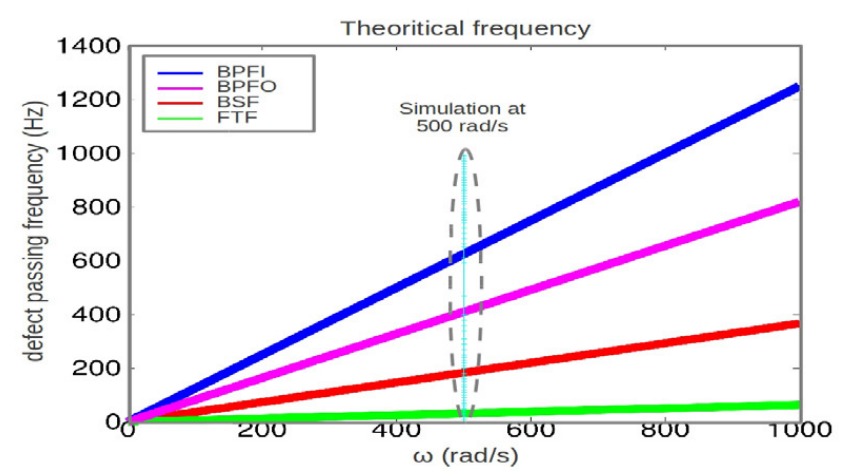

Fig. 10. Theoretical defects frequencies.

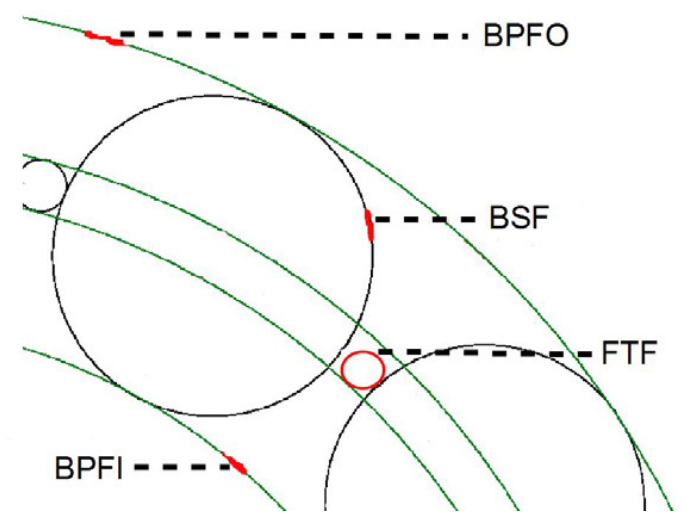

Fig. 11. Consideration of defects.

\section{Simulation of a bearing with defect}

When a bearing is subject to a defect, it fells the defect periodically. This recurring disturbance is visible in the electrical measure. For example, a fault on the outer race will generate a signal containing shocks spaced from the transition period between the balls. Knowing the geometrical characteristics (Tab. 1) and its operating speed $\omega$, it is possible to determine the passage frequencies of various defects, depending on their locations $[1-3]$.

Figure 10 gives us theoretical frequencies for different defects, according to the operation speed. Here, we introduce the next agreement, used to identify bearing defects (Tab. 3 and Fig. 12).

In the remainder of this paper, we postulate that a defect causes a temporary break contact, as suggested in Figure 11. In the next simulations, we control the defect size by defining the rupture contact zone with an angle.

\subsection{Simulation with different defects}

Each proposed defect (Figs. 11 and 12) is simulated for an operating speed $\omega=500 \mathrm{rad} . \mathrm{s}^{-1}$, as healthy results.

Each defect causes disturbances in the electrical measurement. Frequencies determined in Figure 13 are compared with the theory, given by Figure 10. The BSF measured frequency Figure 13d represents the necessary time for the ball defect to encounter successively the two races. Each sudden jump (visible for BPFI, BPFO and BSF) is associated with a contact rupture followed by a shock. The FTF case Figure 13a is likened to the cage clearance, there is no electrical rupture, just a mechanical rupture. This ball/cage contact is insulating. Except for the FTF case, when the defect is not detected, the electrical resistance represents the healthy bearing. We observe a transfer of information between the mechanical configuration and the electrical calculation. In fact, the loss of an electrical contact affects the electrical computation by deleting an electrical way.

\subsection{Simulation with different sizes of the same defect}

Several simulations with defect on the inner race are conducted under the same conditions $(\omega, \mathrm{F})$. We only change the size of the defect, then statistical indicators used for vibrational methods [2] are tested on the data of the electrical resistances.

First, we start by studying the response of the system when the fault grows at $\omega=500$ rad.s ${ }^{-1}$.

As expected, a similar signal is obtained, at the same frequency when the defect is considered (Fig. 14a). The FFT of the healthy signal has no harmonic component, Figure $14 \mathrm{~b}$ and the amplitudes of the harmonics are more pronounced when the defect is larger. As a first approximation, the anomalies due to defect in the electrical resistance can be regarded as a periodic rectangle function. We hope to link these breakthrough times with the size of the defect. Electrical data may be analyzed in time domain by scalar indicators. We consider that the acceleration data in vibration analysis are replaced by the electrical resistance. For a better understanding of the defect size effect on the electrical measurement, we tested the following statistical quantities: Peak, Average, Root Mean Square, Crest Factor, Kurtosis, Shape Factor. In vibrational method, these indicators usually indicate the appearance or the aggravation of a defect. We suppose that the evolution of scalar indicators is more important than their intrinsic value. The scalar indicators could provide informations about machine-related strategic decision. 


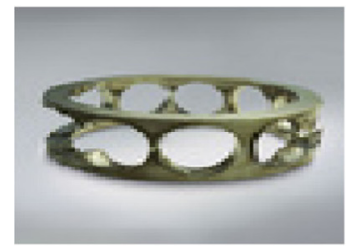

FTF

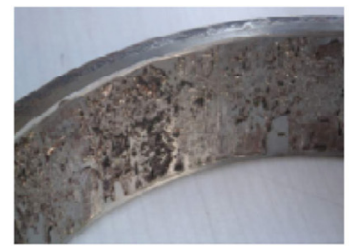

BPFO

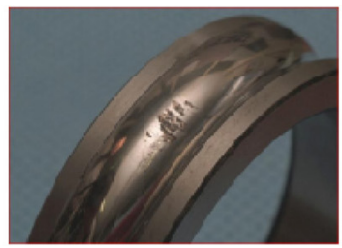

BPFI

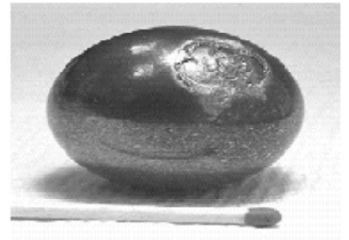

BSF

Fig. 12. Real defect.

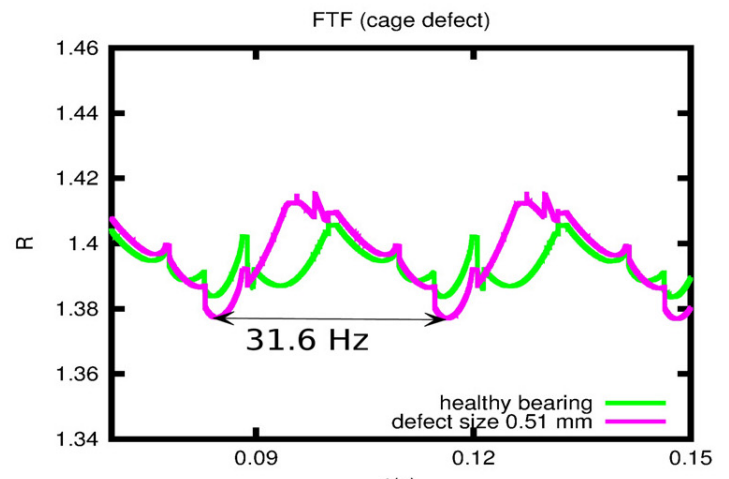

(a)

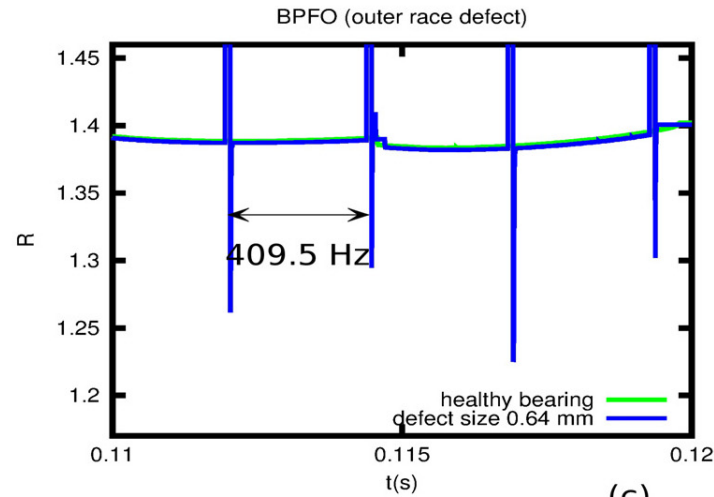

(c)

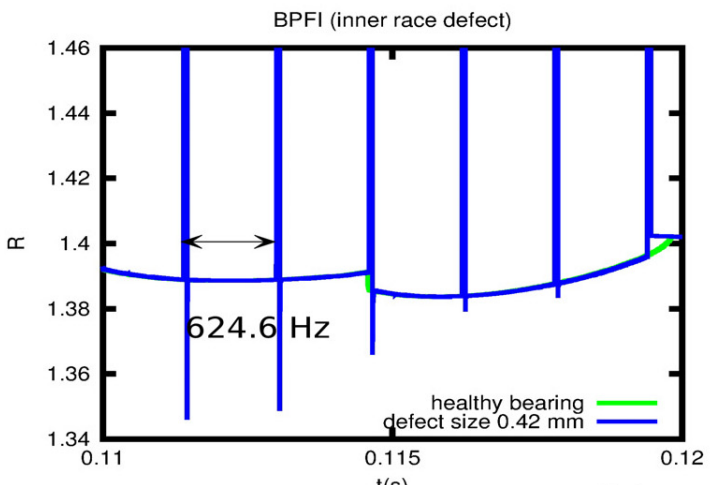

(b)

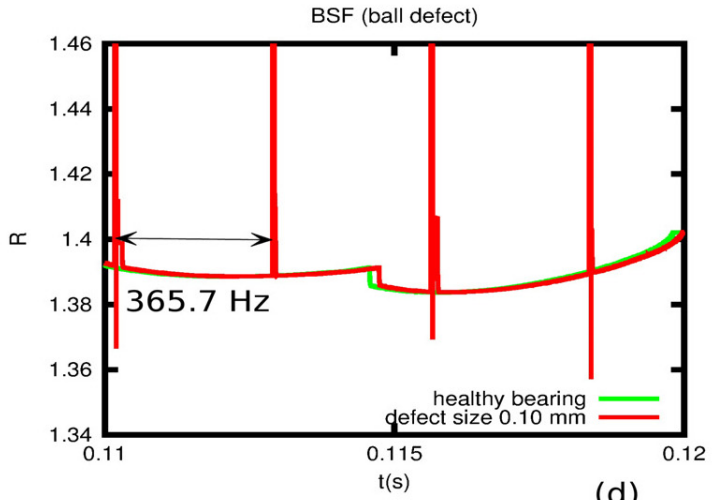

(d)

Fig. 13. Electrical resistance versus time with different defects $\left(\omega=500 \mathrm{rad} . \mathrm{s}^{-1}\right)$.

The following results are calculated over a range of time of $0.1 \mathrm{~s}$, taken out of the sliding phase.

The evolution of the Peak Factor, the Crest Factor and the Shape Factor Figure 15a are not enough sensitive to be able to extract valuable informations. The Average Factor and the Root Mean Square factor Figure 15b show linear variation, as observed in vibration analysis and the two lines are close. The most interesting factor is the kurtosis, presented in Figure 15c, it's sensitive to the damage size.

\section{Conclusion}

Our model correctly reproduced the operation of the ball bearing. The results for the four types of defects provided interesting informations, by comparing the frequencies obtained by numerical simulations with theoretical frequencies. Taking into account the defects by breaking contact appears to be an interesting method. A parametric study for the size of a defect generates a sensitive statistical indicator, the kurtosis, also used in vibrational analysis. The simulations allow to find the effect of a defect in the calculation of the electrical resistance. The transfer of mechanical information to the electrical state is simulated and several parametric studies will soon be conducted. For example, we plan to study the effect of loading conditions and the effect of the rotating speed on the statistical indicators. Meanwhile, an experimental bench will soon complete our digital data. Note that due to the limited sampling frequency measuring devices, it will not be possible to identify defects with a small size, but the expected resolution will be better than vibrational measurements. Furthermore, from the experimental point of view, the electrical measurement is free from the surrounding vibrations which make it easier 

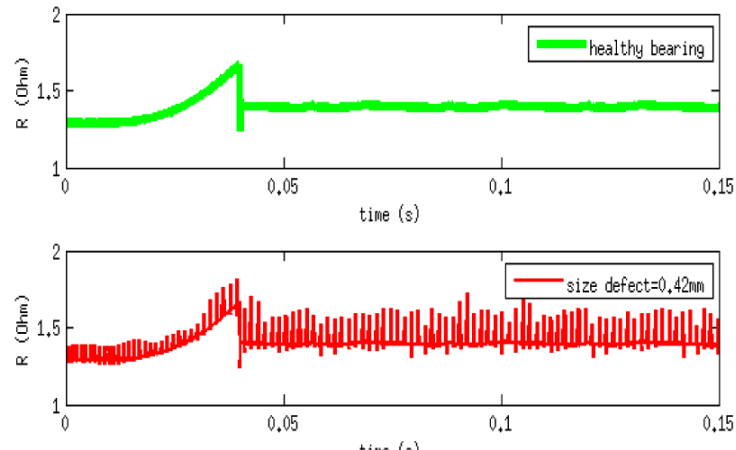

time (s)

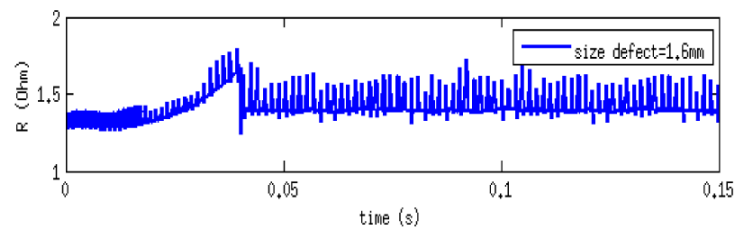

(a)
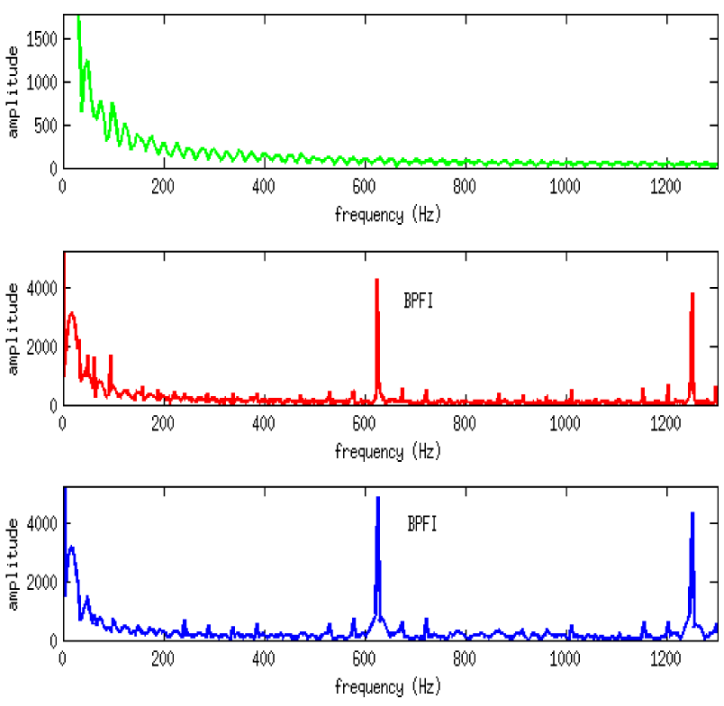

(b)

Fig. 14. (a) Electrical resistance and (b) Fourier transform.
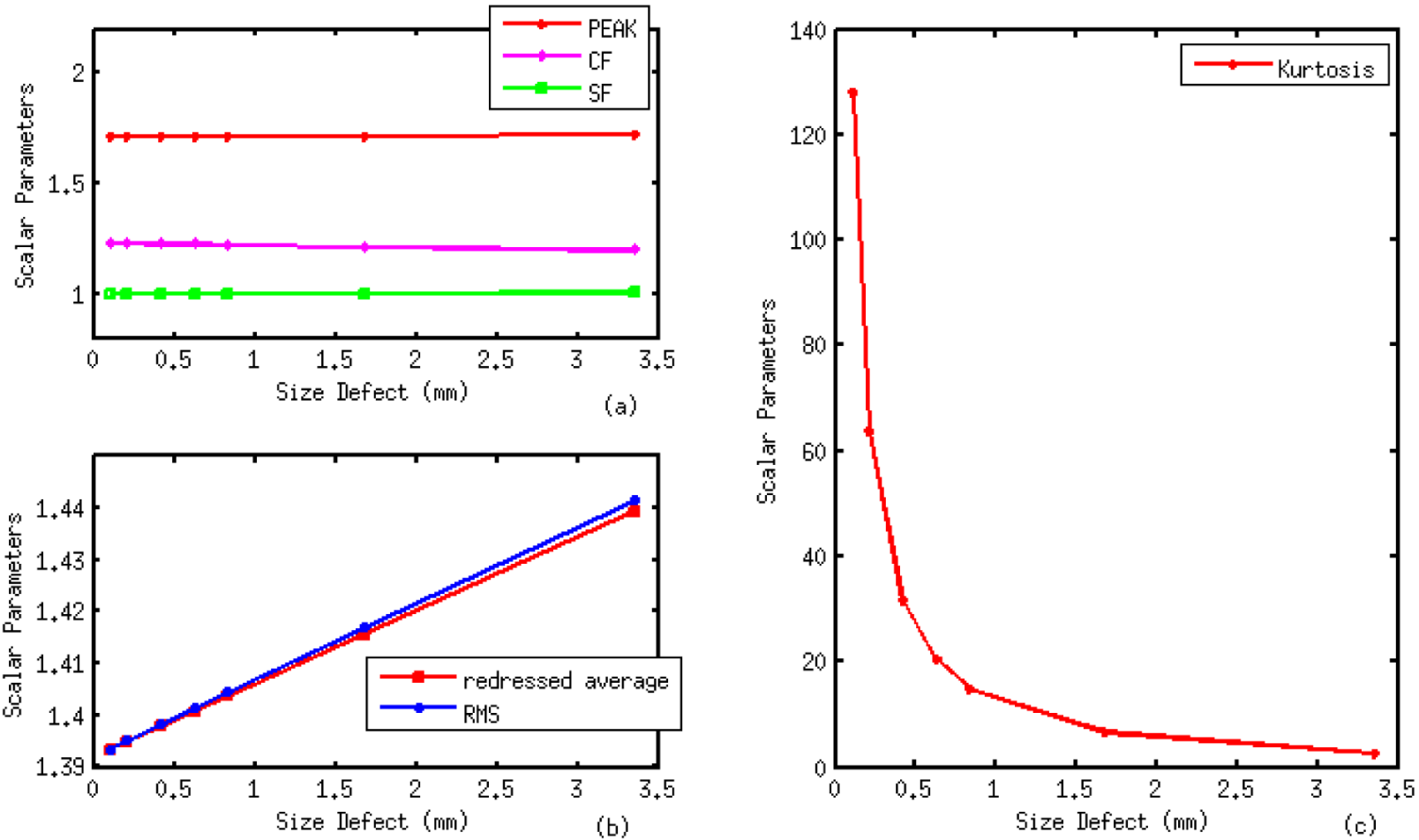

Fig. 15. Evolution of the scalar parameters according to the size of a defect on the inner race.

to implement. Other defects, such as imbalance caused by improper loading of the bearing will be modeled. In the future, we hope to associate each electrical signature to a characteristic defect. All future developments will be led with the best advanced methods of existing approaches.

Acknowledgements. This study has been carried out under project SIGNATELEC (2012-2015). Thanks to the "Région de Picardie" for its financial support.

\section{References}

[1] N. Tandon, A. Choudhury, A review of vibration and acoustic measurement methods for the detection of defects in rolling element bearings, Tribol. Int. 32 (1999) $469-480$

[2] S. Sassi, B. Badri, M. Thomas, Tracking surface degradation of ball bearings by means of new time domain scalar indicators, Int. J. of COMADEM 11 (2008) 36-45 
[3] T. Harris, Rooling Bearing Analysis, Wiley and Sons, 2001

[4] G. De Saxcé, J. Fortin, O. Millet, About numerical simulation of the dynamics of granular media and the definition of the mean stress tensor, Mech. Mater. 36 (2004) $1175-1184$

[5] J. Fortin, O. Millet, G. De Saxcé, Numerical simulation of granular materials by an improved discrete element method, Int. J. Numer. Methods Eng. 62 (2004) 639-663

[6] K. Bourbatache, M. Guessasma, E. Bellenger, V. Bourny, A. Tekaya, Discrete modelling of electrical transfer in multi-contact systems, Granular Matter 14 (2012) 1-10
[7] I. Sanni, E. Bellenger, J. Fortin, P. Coorevits, A reliable algorithm to solve 3D frictional multi-contact problems: application to granular media, J. comput. Appl. Math. 234 (2010) 1161-1171

[8] M. Jean, The non-smooth contact dynamics method, Comput. Methods Appl. Mech. Eng. 177 (1999) 235-257

[9] J.J. Moreau, Some numerical methods in multibody dynamics: application to granular materials, Eur. J. Mech. A Solids 13 (1994) 93-114

[10] J. Greenwood, Analysis of a elliptical Hertzian contact, Tribol. Int. 30 (1997) 235-237 